\title{
One-year follow-up period after transumbilical thoracic sympathectomy for hyperhidrosis: Outcomes and consequences
}

\author{
Li-Huan Zhu, MD, ${ }^{a}$ Quan Du, MD, ${ }^{b}$ Long Chen, MD, ${ }^{a}$ Shengsheng Yang, MD, ${ }^{a}$ Yuanrong Tu, MD, \\ Shengping Chen, $\mathrm{MD},{ }^{\mathrm{a}}$ and Weisheng Chen, $\mathrm{MD}, \mathrm{PhD}^{\mathrm{a}}$
}

\begin{abstract}
Objectives: Thoracic sympathectomy is considered the most effective method to treat palmar hyperhidrosis. We developed a novel approach for thoracic sympathectomy in patients with palmar hyperhidrosis through the umbilicus, using an ultrathin gastroscope. The aim of this study was to evaluate the continuing efficacy and patient satisfaction of this innovative surgery.
\end{abstract}

\begin{abstract}
Methods: All procedures were performed under general anesthesia and the patients were intubated with a dual-lumen endotracheal tube. After a 5-mm umbilical incision, the muscular parts of the diaphragmatic dome were incised with a needle-knife and the nasal gastroscope was advanced into the thoracic cavity. The sympathetic chain was identified at the desired thoracic level and ablated with hot biopsy forceps. All patients were followed up for at least 1 year after the procedure through clinic visits or telephone/e-mail interviews.
\end{abstract}

Results: From April 2010 to August 2011, a total of 35 patients underwent a transumbilical thoracic sympathectomy. Fifty-seven percent were male patients, with a mean age of 21.2 years (range, 16-33 years). The success rate after 12 months was $97.1 \%$ (34 of 35) for isolated palmar hyperhidrosis and $72.2 \%$ (13 of 18) for axillary hyperhidrosis. Compensatory sweating was reported in $28.6 \%$ of patients at the 1 -year follow-up evaluation. There was no mortality, no diaphragmatic hernia, and no Horner syndrome was observed. Quality of life related to hyperhidrosis improved substantially in $27(77.1 \%)$ patients, and improved in 4 $(11.4 \%)$ patients at 12 months after surgery. A total of $94.3 \%$ of patients were satisfied with the excellent cosmetic results of the surgical incision.

Conclusions: Transumbilical thoracic sympathectomy is an efficacious alternative to the conventional approach. This technique avoided the chronic pain and chest wall paresthesia associated with the chest incision. In addition, this novel procedure afforded maximum cosmetic benefits. (J Thorac Cardiovasc Surg 2014;147:25-9)

Primary hyperhidrosis is a disabling disorder of excessive sweating that usually begins in either childhood or adolescence. The sites most commonly affected are the palms, soles, and axillae. This condition is fairly common and affects up to $4.59 \%$ of the population in southern China. ${ }^{1}$ Although hyperhidrosis does not pose a serious health threat, it may have serious social, emotional, and professional consequences, and negatively impact quality of life.

Thoracoscopic sympathetic ganglion resection or ablation via 2 to 4 incisions on the chest is the only effective

\footnotetext{
From the Department of Cardiothoracic Surgery, ${ }^{a}$ Fuzhou General Hospital, Fujian Medical University, Fuzhou, People's Republic of China; and Department of Cardiothoracic Surgery, ${ }^{\text {b }}$ The First Clinical Medical College of Fujian Medical University, Fuzhou, People's Republic of China.

Supported by the Key Project of Science and Technology of Fujian province (grant 2010I0011).

Disclosures: Authors have nothing to disclose with regard to commercial support. Li-Huan Zhu and Quan Du contributed equally to this paper.

Read at the 93rd Annual Meeting of The American Association for Thoracic Surgery, Minneapolis, Minnesota, May 4-8, 2013.

Received for publication April 5, 2013; revisions received July 17, 2013; accepted for publication Aug 27, 2013; available ahead of print Oct 30, 2013.

Address for reprints: Weisheng Chen, MD, PhD, No. 156 West Second Ring Rd, Gulou District, Fuzhou 350001, People's Republic of China (E-mail: weishengmd@hotmail.com).

$0022-5223 / \$ 36.00$

Copyright (c) 2014 by The American Association for Thoracic Surgery

http://dx.doi.org/10.1016/j.jtcvs.2013.08.062
}

and sustainable surgical method for the treatment of primary hyperhidrosis. ${ }^{2,3}$ This procedure can be performed through a single $10-\mathrm{mm}$ port on each side of the chest with a 5-mm thoracoscope. It also can be performed with a needle scope, which generally requires 2 or 3 small ports on each side of the chest. However, chronic postoperative problems caused by damage to the intercostal nerve during thoracoscopic surgery were present in $31.4 \%$ of patients in a previous study. ${ }^{4}$ The symptoms include pain, numbness, and paresthesia. To avoid these disadvantages, we conducted a study on embryonic natural orifice transumbilical endoscopic surgery (E-NOTES), which eliminates the need for chest incisions. The early results showed that E-NOTES sympathectomy was technically feasible and safe in the treatment of primary hyperhidrosis, ${ }^{5}$ but continuing efficacy wais unknown. The purpose of this study was therefore to assess the clinical results of patients who had a follow-up period of at least 1 year after the time of surgery and to evaluate the durability of this surgical approach for hyperhidrosis.

\section{PATIENTS AND METHODS}

Between April 2010 and August 2011, all patients who underwent a transumbilical thoracic sympathectomy for primary palmar hyperhidrosis $(\mathrm{PH})$ with or without primary axillary hyperhidrosis $(\mathrm{AH})$ in our institution 


$\begin{array}{ll}\text { Abbreviations and Acronyms } \\ \text { AH } & =\text { axillary hyperhidrosis } \\ \text { CS } & =\text { compensatory sweating } \\ \text { E-NOTES }= & \text { embryonic natural orifice } \\ & \text { transumbilical endoscopic surgery } \\ \text { NOTES } & =\text { natural orifice translumenal endoscopic } \\ & \text { surgery } \\ \text { PH } & =\text { palmar hyperhidrosis } \\ \text { QOL } & =\text { quality of life } \\ \text { VAS } & =\text { visual analog scales } \\ \text { VATS } & =\text { video-assisted thoracic surgery }\end{array}$

were included in a prospective follow-up study evaluating the results of their surgery.

Criteria for inclusion in the study were severe primary PH that significantly interfered with daily life and work. Criteria for exclusion were the secondary hyperhidrosis caused by hyperthyroidism; neurotic anxiety; the existence of diseases such as cardiac diseases, pulmonary infections, pleural or peritoneal diseases that could increase surgical risk; or patients who were a high risk for general anesthesia. Patients who had less than 1 year of follow-up evaluation were excluded.

The study protocol was accepted by the institutional review board, and informed consent was obtained from all patients.

\section{Surgical Technique}

All procedures were performed under general anesthesia with double-lumen endotracheal intubation. Patients were placed in a $30^{\circ}$ reverse-Trendelenburg position with the arms abducted. A 5-mm incision was made within the umbilicus, and a homemade long trocar (length, 60 $\mathrm{cm}$; internal diameter, $5.8 \mathrm{~mm}$; and wall thickness, $0.25 \mathrm{~mm}$ ) was inserted as a guide for a nasal gastroscope (GIF-XP260N; Olympus Medical Systems, Tokyo, Japan). The trocar also was used to establish pneumoperitoneum, which was achieved by insufflating the abdominal cavity with carbon dioxide at $10 \mathrm{~mm} \mathrm{Hg}$. The anesthetized patients were tilted $30^{\circ}$ to the left. The site of transdiaphragmatic approach was chosen carefully on the muscular pars of the right diaphragmatic dome. Under the left-lung unilateral ventilation, a 5-mm incision was made with a needle-knife (Alton Medical Equipment C., Ltd, Shanghai, China). Subsequently, the needle-knife served as a guidewire for insertion of the endoscope into the right thoracic cavity while the pneumoperitoneum was released. Hot biopsy forceps (Alton Medical Equipment C., Ltd) were used to grasp and ablate the T3 ganglia for $\mathrm{PH}$, and the T3+T4 ganglion for patients who had combined $\mathrm{PH}$ and $\mathrm{AH}$. If accessory fibers and the Kuntz nerve were found, they were cauterized. A palmar temperature increase of $1.5^{\circ} \mathrm{C}$ confirmed adequate sympathectomy. Then the right lung was reinflated when the nasal gastroscope was withdrawn to the abdominal cavity. The operating table then was tilted $30^{\circ}$ to the right. The procedure was performed on the left side in a similar manner. After suctioning the remaining air in the abdominal cavity, the nasal gastroscope was withdrawn from the abdominal cavity and the umbilical incision was closed with skin glue.

\section{Follow-up Evaluation and Data Collection}

The demographic details, surgical time, intraoperative complications, immediate improvement of symptoms, early postoperative complications, and postoperative hospital stay data were collected from the medical records.

All patients were routinely followed up with an office visit at 1 day, 3 days, and 1 week after surgery. The pain scores were evaluated using visual analog scales (VAS) from 0 (no pain) to 10 (worst pain ever experienced).

The long-term data were collected through clinic visits or telephone/ e-mail interviews at 12 months after surgery. Patients were required to fill out a detailed questionnaire that included resolution of symptoms, postoperative complications, levels of satisfaction with the procedure, incidence of symptom recurrence, sweating symptom-related quality of life (QOL) after the procedure, and satisfaction with the aesthetic result.

\section{RESULTS}

A total of 35 patients treated by transumbilical thoracic sympathectomy for primary $\mathrm{PH}$ (or with $\mathrm{AH}$ ) were the subject of this study. There were 20 male patients $(57.1 \%)$ and 15 female patients $(42.9 \%)$, with an average age of 21.2 years (range, 16-33 years). Seventeen patients $(48.6 \%)$ had $\mathrm{PH}$ only and 18 patients $(51.4 \%)$ had $\mathrm{PH}$ and $\mathrm{AH}$ (Table 1).

Bilateral thoracic sympathectomies were successful in all patients. The anatomic level of E-NOTES sympathectomy was $\mathrm{T} 3$ in 17 patients and T3+T4 in 18 patients, resulting in immediate resolution of sweating symptoms after surgery. The mean surgical time was $65 \pm 17$ minutes (range, 47-105 min). There was no surgical mortality or major intraoperative complication. No patients required conversion to thoracoscopic surgery or thoracotomy. There were 2 cases $(5.7 \%)$ of residual pneumothorax during the immediate postoperative period, which did not require pleural drainage. All patients were discharged from the hospital the following day. The average VAS-based pain score measured at days 1 and 3 after surgery was $1.43 \pm 0.51$ and $0.06 \pm 0.23$, respectively. At day 1 after surgery, 4 patients reported incision site numbness, which disappeared 1 week after surgery (Table 2).

Follow-up evaluations were $100 \%$ complete at a median of 14 months, and a range of 12 to 18 months. There was no mortality, no diaphragmatic hernia, and no Horner syndrome was observed. The success rate was $97.1 \%$ (34 of 35) for $\mathrm{PH}$ and $72.2 \%$ (13 of 18) for $\mathrm{AH}$. No intractable hyperhidrosis was found in this series of patients. $\mathrm{PH}$ recurred in 1 patient $(2.9 \%)$ during the followup period. Thirty-three patients $(94.3 \%)$ were satisfied with the excellent cosmetic results of the surgical incision.

Compensatory sweating (CS) occurred in 10 patients $(28.6 \%)$. In 8 cases $(22.9 \%)$ it appeared before 6 months and in 2 cases $(5.7 \%)$ it appeared between 6 and 12 months. Seven cases $(20 \%)$ were classified as mild, 2 cases $(5.7 \%)$ were classified as moderate, and 1 case $(2.9 \%)$ was classified as severe. The most frequent locations were the back $(56 \%)$, abdomen $(51 \%)$, lower extremities $(45 \%)$, and thorax $(8.6 \%)$. Over time, 4 patients $(11.4 \%)$ reported improvement, $5(14.3 \%)$ had no change, and 1 patient $(2.9 \%)$ got worse.

Sweating symptom-related QOL after the E-NOTES sympathectomy procedure was quantified in responders 
TABLE 1. Characteristics of patients undergoing transumbilical thoracic sympathectomy

\begin{tabular}{lc}
\hline \multicolumn{1}{c}{ Demographics } & Patients, $\mathbf{n}(\%)$ \\
\hline Total patients & 35 \\
Median age (range), y & $21.2(16-33)$ \\
Female sex & $15(42.9)$ \\
Affected hyperhydrotic area & \\
Hands alone & $17(48.6)$ \\
Hands and axilla & $18(51.4)$ \\
\hline
\end{tabular}

through the use of a questionnaire. Of a total of 35 patients, 27 patients $(77.1 \%)$ stated they were very satisfied, 4 patients $(11.4 \%)$ stated they were somewhat satisfied, 2 patients $(5.7 \%)$ stated no change, and 1 patient $(2.9 \%)$ stated they regretted having had the surgery (Table 3 ).

\section{DISCUSSION}

Primary hyperhidrosis has no known cause, but inheritance is a risk factor and $15.3 \%$ of patients have a family history of the disorder. ${ }^{1}$ A variety of treatment modalities are used to reduce sweating in people suffering from hyperhidrosis. Nonsurgical treatments consist of topical aluminum chloride, oral anticholinergics, ionotophoresis, and botulinum toxin A (Botox; Allergan, Inc, Irvine, Calif) injections. ${ }^{6}$ However, none of these methods have a durable clinical outcome. The main secondary effect of aluminum chloride is skin irritation. The effects of botulinum toxin A injections last only 3 to 9 months. The process of ionotophoresis is time consuming.

Thoracoscopic sympathectomy is considered the gold standard for the treatment of palmar hyperhidrosis. Nevertheless, it is reported that up to $25 \%$ of patients complained of sustained thoracic numbness and pain after thoracoscopy. ${ }^{7}$ Even with the use of needlescopic

TABLE 2. Surgical details of patients undergoing transumbilical thoracic sympathectomy

\begin{tabular}{lc}
\multicolumn{1}{c}{ Demographics } & Result \\
\hline Level of sympathectomy, n $(\%)$ & $17(48.6)$ \\
T3 & $18(51.4)$ \\
T3+T4 & \\
Surgical details, mean \pm SD (range) & $17(47-105)$ \\
$\quad$ Surgical time, min & 1 \\
Postoperative hospital stay, d & $2(5.7)$ \\
Complication immediately postoperatively, n $(\%)$ & $1.43 \pm 0.51$ \\
$\quad$ Pneumothorax & $0.06 \pm 0.23$ \\
VAS pain scores, mean \pm SD & \\
1 d after surgery & $4(11.4)$ \\
3 d after surgery & $0(0.0)$ \\
Paresthesia distinct from wound pain, $\mathrm{n}(\%)$ & \\
1 d after surgery & \\
1 wk after surgery & \\
\hline T3, Third thoracic level; $T 4$, fourth thoracic level; $S D$, standard deviation; $V A S$, visual \\
analog scales.
\end{tabular}

TABLE 3. The follow-up questionnaire and outcomes

\begin{tabular}{lc}
\hline \multicolumn{1}{c}{ Demographics } & Result \\
\hline Surgical results, $\mathrm{n}(\%)$ & \\
PH complete resolution & $34(97.1)$ \\
AH complete resolution & $13(72.2)$ \\
PH recurred & $1(2.9)$ \\
Compensatory sweating, n (\%) & \\
Mild & $7(20)$ \\
Moderate & $2(5.7)$ \\
Severe & $1(2.9)$ \\
Quality of life related to hyperhidrosis, $\mathrm{n}(\%)$ & \\
Much better & $27(77.1)$ \\
Slightly better & $4(11.4)$ \\
The same & $2(5.7)$ \\
Slightly worse & $1(2.9)$ \\
Much worse & $1(2.9)$ \\
Satisfaction of aesthetic result, $\mathrm{n}(\%)$ & $33(94.3)$ \\
\hline$P H$, Palmar hyperhidrosis; $A H$, axillary hyperhidrosis.
\end{tabular}

video-assisted thoracic surgery (VATS), $12.9 \%$ of the patients still experienced shoulder joint dysfunction on day 1 after surgery and $19.4 \%$ of the patients still had chest wall paresthesia around the incisions 7 days after surgery. ${ }^{8}$ The main explanation for these problems was the trauma to the intercostal nerve when the trocars were introduced into the pleural cavity.

To find surgical procedures that avoid chest incisions and reduce the number of ports, Turner et $\mathrm{al}^{9}$ and Yang et $\mathrm{al}^{10}$ conducted an animal study using the technique of NOTES to perform endoscopic thoracic sympathetic trunk resection via the esophagus or oral vestibular incision. Despite the substantial work in the NOTES field, it is clear that there is a long way to go from the present animal experiments to clinical practice.

An alternative and competing technology to NOTES is E-NOTES, which also is called single-incision laparoscopic surgery in the field of laparoscopic surgery. Several studies have shown that this technique is less invasive than conventional 3-port surgeries and is safer than NOTES..$^{11-13}$ Wen et $\mathrm{al}^{14}$ reported that the transumbilical approach would obviate the potential complications of intercostal nerve injury, neuralgia, and chronic thoracic pain.

We began with clinical trials of E-NOTES sympathectomy for palmar hyperhidrosis in April 2010. The short-term follow-up evaluation confirmed that this technique was feasible and safe. A VAS pain score assessment showed that incision-related pain largely disappeared 3 days after surgery and there was no incision-related discomfort 1 week after surgery, which partially could be attributed to the absence of intercostal nerve injury. One-year follow-up results for the patients showed that the transumbilical endoscopic thoracic sympathectomy was an effective and durable treatment for hyperhidrosis. Patient satisfaction and perceived 
effectiveness of sympathectomy for palmar or axillary hyperhidrosis remain high even 1 year after the procedure. A number of clinical studies reported that conventional VATS thoracic sympathectomies yielded a success rate of greater than $95 \%$ in the treatment of primary hyperhidrosis. ${ }^{15-17}$ Our results are in keeping with these reports, with $96 \%$ of patients reporting substantial improvement of their $\mathrm{PH}$ and $72.2 \%$ of patients reporting disappearance of their AH. A total of $88.5 \%$ of patients showed a significant improvement in QOL after the procedure. Moreover, surgical incisions have been reduced from 4 in traditional techniques to 1 hidden in the umbilicus, leading to very favorable cosmetic benefits.

The most common secondary effect of thoracic sympathectomy is CS, which occurs soon after the surgery or many years later. The most affected areas are the upper and lower back, lower chest, abdomen, buttocks, groin, and backs of the thighs. The severity of CS can be divided into mild CS, moderate CS, and severe CS. ${ }^{18}$ Mild CS creates moisture on the body, but does not penetrate through clothing. Moderate CS involves moisture that can show through clothes. Severe CS causes profuse sweating that stains one's shirts and pants. The reported frequencies of CS vary considerably. Licht and Pilegaard ${ }^{18}$ reported an incidence of CS in $89 \%$ of patients in a series of 158 patients, and in $35 \%$ of patients it was so severe that they often had to change their clothes during the day. However, $\mathrm{Li}$ et $\mathrm{al}^{19}$ reported the incidence of mild, moderate, and severe CS as only $13 \%, 8 \%$, and $6 \%$, respectively. A total of $28.6 \%$ of our patients developed CS by their 1-year follow-up evaluation, which is similar to the result of found in the study by $\mathrm{Li}$ et al. ${ }^{19}$ The mechanism of CS is unknown. Deng et $\mathrm{al}^{20}$ reported that the higher level sympathectomy interrupted the higher rate of CS happened, and the more destructive the procedure, the higher the risk of compensatory sweating.

This technique is still relatively challenging and requires longer surgical time, and it also takes time for thoracic surgeons to become familiar with gastroscopy. The limitations of this study were the small volume of patients and the lack of randomized clinical trials. Although there was no evidence of thoracic or abdominal organ injury during the surgery, the technique potentially increased the risk of intra-abdominal tissue injury.

\section{CONCLUSIONS}

Follow-up results show that E-NOTES yields a comparable cure rate with VATS for hyperhidrosis. In view of the unique characteristics of the surgical approach, we recommend the procedure as a new treatment option for the following patients with $\mathrm{PH}$ : patients who have a past history of breast augmentation and patients who do not want to have visible incisions on their chest.

\section{References}

1. Tu YR, Li X, Lin M, Lai FC, Li YP, Chen JF, et al. Epidemiological survey of primary palmar hyperhidrosis in adolescent in Fuzhou of People's Republic of China. Eur J Cardiothorac Surg. 2007;31:737-9.

2. Ng CS, Yeung EC, Wong RH, Kwok MW. Single-port sympathectomy for palmar hyperhidrosis with Vasoview Hemopro 2 endoscopic vein harvesting device. J Thorac Cardiovasc Surg. 2012;44:1256-7.

3. Miller DL, Bryant AS, Force SD, Miller JI Jr. Effect of sympathectomy level on the incidence of compensatory hyperhidrosis after sympathectomy for palmar hyperhidrosis. J Thorac Cardiovasc Surg. 2009;138:581-5.

4. Liu YH, Liu HP, Wu YC, Ko PJ. Feasibility of transtracheal surgical lung biopsy in a canine animal model. Eur J Cardiothorac Surg. 2010;37:1235-6.

5. Zhu LH, Wang W, Yang S, Li D, Zhang Z, Chen S, et al. Transumbilical thoracic sympathectomy with an ultrathin flexible endoscope in a series of 38 patients. Surg Endosc. 2013;27:2149-55.

6. Walling HW, Swick BL. Treatment options for hyperhidrosis. Am J Clin Dermatol. 2011;12:285-95.

7. Steegers MA, Snik DM, Verhagen AF, van der Drift MA, Wilder-Smith OH. Only half of the chronic pain after thoracic surgery shows a neuropathic component. J Pain. 2008;9:955-61.

8. Sihoe AD, Cheung CS, Lai HK, Lee TW, Thung KH, Yim AP. Incidence of chest wall paresthesia after needlescopic video-assisted thoracic surgery for palmar hyperhidrosis. Eur J Cardiothorac Surg. 2005;27:313-9.

9. Turner BG, Gee DW, Cizginer S, Konuk Y, Karaca C, Willingham F, et al. Feasibility of endoscopic transesophageal thoracic sympathectomy (with video). Gastrointest Endosc. 2010;71:171-5.

10. Yang C, Chu Y, Wu YC, Hsieh MJ, Lu MS, Liu CY, et al. The lateral decubitus position improves transoral endoscopic access to the posterior aspects of the thorax. Surg Endosc. 2012;26:2988-92.

11. Vilallonga R, Barbaros U, Sümer A, Demirel T, Fort JM, González O, et al. Single-port transumbilical laparoscopic cholecystectomy: a prospective randomised comparison of clinical results of 140 cases. J Minim Access Surg. 2012;8:74-8.

12. Vilallonga R, Barbaros U, Nada A, Sümer A, Demirel T, Fort JM, et al. Singleport transumbilical laparoscopic appendectomy: a preliminary multicentric comparative study in 87 patients with acute appendicitis. Minim Invasive Surg. 2012;2012:492409.

13. Abe N, Takeuchi H, Ueki H, Yanagida O, Masaki T, Mori T, et al. Single-port endoscopic cholecystectomy: a bridge between laparoscopic and translumenal endoscopic surgery. J Hepatobiliary Pancreat Surg. 2009;16:633-8.

14. Wen CT, Chu Y, Yeh CJ, Liu CY, Yuan HC, Ko PJ, et al. Feasibility and safety of endoscopic transumbilical thoracic surgical lung biopsy: a survival study in a canine model. J Surg Res. 2013;183:47-55.

15. Sugimura H, Spratt EH, Compeau CG, Kattail D, Shargall Y. Thoracoscopic sympathetic clipping for hyperhidrosis: long-term results and reversibility. J Thorac Cardiovasc Surg. 2009;137:1370-6.

16. Rodríguez PM, Freixinet JL, Hussein M, Valencia JM, Gil RM, Herrero J, et al. Side effects, complications and outcome of thoracoscopic sympathectomy for palmar and axillary hyperhidrosis in 406 patients. Eur J Cardiothorac Surg. 2008;34:514-9.

17. Yanagihara TK, Ibrahimiye A, Harris C, Hirsch J, Gorenstein LA. Analysis of clamping versus cutting of T3 sympathetic nerve for severe palmar hyperhidrosis. J Thorac Cardiovasc Surg. 2010;140:984-9.

18. Licht PB, Pilegaard HK. Severity of compensatory sweating after thoracoscopic sympathectomy. Ann Thorac Surg. 2004;78:427-31.

19. Li X, Tu YR, Lin M, Lai FC, Chen JF, Miao HW. Minimizing endoscopic thoracic sympathectomy for primary palmar hyperhidrosis: guided by palmar skin temperature and laser Doppler blood flow. Ann Thorac Surg. 2009;87:427-31.

20. Deng B, Tan QY, Jiang YG, Zhao YP, Zhou JH, Ma Z, et al. Optimization of sympathectomy to treat palmar hyperhidrosis: the systematic review and meta-analysis of studies published during the past decade. Surg Endosc. 2011; 25:1893-901.

\section{Discussion}

Dr Vivek Rao (Toronto, Ontario, Canada). Have you done other procedures other than hyperhidrosis through the transumbilical approach?

Dr Chen. No. 
Dr Sergei Mitnovetski (Toronto, Ontario, Canada). Dr Chen, I still do not see why you would prefer this approach. We know that thoracoscopic sympathectomy is an effective operation and the level of complication is pretty low to do it thoracoscopically. Why would you bother to go through an umbilical approach?

Dr Chen. Sorry, can you repeat the question?

Dr Matthew Williams (New York, NY). The question is, the thoracoscopic approach works well with a low complication rate, so how do you justify using this approach as opposed to the standard?

Dr Chen. We usually do the conventional thoracoscopic sympathectomy, but we find some patients suffer from chest incision pain, so I want to avoid the chest incision and find a new approach.
Dr Matthew Williams. I think it is very innovative. How do you close the diaphragm?

Dr Chen. It is very small. The size is just $5 \mathrm{~mm}$, so it is too small to cause any hernia. So you do not need to close the incision of the diaphragm.

Dr Osman Al-Radi (Jeddah, Saudi Arabia). The phrenic nerve is visible only from the thoracic side of the diaphragm When you are puncturing the diaphragm from below, have you had any problems with injuring the phrenic nerve or diaphragmatic paralysis?

Dr Chen. Thank you for your question. The incision of the diaphragm is far from the center of the diaphragm, so I do not think it will cause injury of the nerve. 